\title{
LA TRANSVERSALIDAD CURRICULAR, UNA DÉCADA DESPUÉS
}

\section{CURRICULA TRANSVERSALLITY, ONE DECADE AFTERWARDS}

\author{
Narciso García Nieto*, Rafael Carballo**, Ma José Fernández, Díaz*** \\ Universidad Complutente
}

\begin{abstract}
RESUMEN
El presente artículo pretende revivir o replantear, a más de una década de su implantación, la vigencia y transcendencia de la Transversalidad Curricular. Tema que, en su origen, suscitó una gran expectación y polémica entre los educadores, pero que, actualmente, parece un tanto olvidado o relegado a un segundo plano. Sin embargo, hechos de la más reciente actualidad como el aumento precoz de consumo de alcohol («botellón»), tabaco, drogas, accidentes juveniles de tráfico, etc. están pidiendo imaginación y esfuerzo a la educación, por ser un problema del que la escuela no puede desentenderse y, tal vez, uno de los medios más eficaces de prevención.

Además de hacer una llamada a la sensibilidad y responsabilidad de los educadores y psicopedagogos, el trabajo pretende ofrecer reflexiones y pautas de actuación para llevar a cabo programas de intervención, sobre todo, en lo referente a la «educación para la salud», dentro de los colegios o instituciones educativas.
\end{abstract}

Palabras clave: Transversalidad curricular, educación para la salud, programas de intervención

\begin{abstract} teachers, ...).

\footnotetext{
** Profesor Titular, Facultad de Educación. Universidad Complutense.

***Profesora Titular, Facultad de Educación. Universidad Complutense.
}

* Catedrático, Facultad de Educación. Universidad Complutense.
\end{abstract}

This article try to remember, after more than a decade from its implementation, the relevance and actuality of the «Curricular Transversality». This issue, in its genesis, awoke a great expectation and polemic among educators, but nowadays it seems to be forgotten. However, some actual facts like the precocious increment of alcohol, cigarettes and drugs consumers, the huge number of teenager traffic accidents, etc., are asking to our Educative System for imagination and effort; cause schools cannot obviate these problems for being one of the most appropriate environments to treat them (counselors,

The present work offers thoughts and criteria to act and to elaborate intervention programs, mainly about «Health Education», cause it seems not to be developed with the seriousness and motivation 
which it needs. Furthermore it offers and justifies the bases and references that have to be considered in the daily reality of the Educational Institutions or Schools.

Key words: Curricular Transversality, Health Education, Intervention Programs.

\section{Introducción}

Uno de los temas que más interés y polémica suscitaron con la implantación de la LOGSE, fueron los denominados temas o ejes transversales. Suponían una novedad curricular que despertaron la curiosidad y perplejidad en muchos medios educativos y de no pocos docentes. Aparecieron profusamente aportaciones teóricas, formas y alternativas de tratamiento, programas para poder afrontarlos, etc. No fueron menores las quejas frecuentes, las críticas mordaces y oposiciones frontales a la novedad que tal temática ofrecía.

Situados ya a más de una década de distancia de aquel turbulento origen, nos parece observar que el mencionado problema se silencia hasta tal punto que no sólo no se habla de él sino que parece que no existiera. Ante esta realidad, una docena de años más tarde nos preguntamos: ¿Qué fue de todo aquello? ¿Qué se ha hecho con la denominada transversalidad curricular? ¿Cómo se está afrontando? ¿Supuso una tormenta, momentánea, que el tiempo se ha encargado desvanecer?

Nos parece importante retomar o revisar algo que juzgamos de suma trascendencia y que supone uno de los aspectos más característicos de la última reforma educativa y sobre lo que ha de incidir la que está en marcha. El actual, «tema del botellón», controvertido y polémico, relacionado con la educación para la salud; el consumo de tabaco; la alarmante y creciente generalización en su consumo, entre los jóvenes, de las drogas de diseño o de otro tipo; los frecuentes accidentes de tráfico; ... Son otros tantos problemas actuales que están, inexorablemente, interrogando a la educación y a la escuela.

Este trabajo pretende revivir y sensibilizar a los educadores con la decisiva importancia, individual, sociocomunitaria y educativa, que tiene la transversalidad curricular, a la que hace algún tiempo se le sobrevaloró y hoy nos tememos que esté relegada a un segundo plano o, tal vez, olvidada.

\section{Relevancia de la transversalidad curricular}

En nuestra opinión, la sustantividad de los Temas Transversales reside en que se refieren a problemas o realidades candentes, pertenecientes al ámbito social y que son de máxima transcendencia, a la vez, para el individuo y para la sociedad. Tienen como punto de mira dimensiones o aspectos de la vida humana que implican profunda, vital y existencialmente al individuo y a la sociedad en un momento o situación coyuntural dada, requiriendo un tratamiento y una respuesta educativa adecuada. Olvidar esto supondría no educar ni desde la vida ni para la vida. Lo que significaría una abierta ruptura entre lo que la escuela hace y lo que el alumnado demanda y necesita. Sería muy lamentable que desde la educación no se intentara responder adecuadamente a la problemática surgida en un tiempo y lugar determinados de los que el alumno proviene y en los que debe ser insertado sin mayores riesgos ni para él ni para la sociedad. Creemos que siguen siendo válidos los argumentos justificativos de la transversalidad esgrimidos por diferentes expertos: 
En opinión de González Lucini (1994) los ejes o temas transversales son: «Realidades o necesidades que tienen una especial relevancia para la vida de las personas y para la positiva y armónica construcción de la sociedad contemporánea».

Por su parte, el M.E.C. (1993) en el Diseño Curricular Base, al referirse a los Temas Transversales no duda en afirmar que:

«Se trata de contenidos educativos valiosos, que responden a un proyecto válido de sociedad y de educación, y que, por consiguiente, están plenamente justificados dentro del marco social en el que ha de desarrollarse toda la educación. Son aspectos de especial relevancia para el desarrollo de la sociedad en relación con el consumo, la igualdad, la paz, el medio ambiente, la salud, el ocio, etc.»

Son temas que inciden y entroncan con una base ética, social y personal, referida a intereses y valores comunes, más o menos universales, relacionándose muy estrechamente con el mundo de la axiología y las actitudes, estando estrechamente relacionados con los problemas de la sociedad contemporánea «siendo valores importantes tanto para el desarrollo personal e integral de los alumnos y alumnas, cuanto para un proyecto de sociedad más libre y pacífica y más respetuosa hacia las personas y hacia la propia naturaleza que constituye el entorno de la sociedad humana», González Lucini (1994).

Alvarez Martín (1994) concibe los temas transversales como:

"Aquellos aspectos fundamentales en la vida cotidiana presentes en el curriculum escolar a través de las diferentes áreas, formando parte de sus contenidos y que deben ser uno de los ejes vertebradores de la actividad docente».

Vemos en la transversalidad un elemento y esfuerzo, plausibles, para intentar trazar un horizonte educativo común para todos los ciudadanos que viven en una sociedad democrática, como la actual, suponiendo unos objetivos formativos que impulsan y favorecen un desarrollo ético y unos comportamientos humanos y humanizadores, sin los cuales la sociedad no sólo no se desarrolla convenientemente sino que hasta corre el riesgo de empobrecerse o aniquilarse. La escuela no puede ser insensible a este encomiable objetivo, difícil de conseguir si los educadores no se lo toman muy en serio

Una educación que se desentienda de las interrogaciones, los problemas reales, profundos, de los alumnos y que no dé respuesta a sus necesidades más acuciantes no puede satisfacer ni a los alumnos ni a los padres ni a los mismos educadores. Nuestra sociedad es tan compleja y disfuncional, con tal capacidad de influencia y manipulación, que puede hacer de los niños y de los adolescentes unas fáciles víctimas del consumismo, la superficialidad, los intereses de grupos e ideologías, tendentes a imponer unas normas y unos estilos de vida más propios de una jerarquía de seudovalores capaces de provocar comportamientos inadecuados y deshumanizadores.

La transversalidad curricular puede ser un buen antídoto contra todos estos males. Es un esfuerzo por fortalecer la personalidad de los alumnos, procurando suscitar en ellos una capacidad de análisis y crítica de cuanto a su alrededor sucede para adoptar criterios acertados, actitudes responsables y un compromiso con todo lo que favorezca una vida personal y una sociedad saludable y humanizadora. En el fondo de este trabajo está la intencionalidad de replantear ante los educadores las preguntas anteriormente formuladas, intentando devol- 
ver a la transversalidad curricular el papel que le corresponde dentro del actual curriculum y del quehacer de la escuela actual.

No cabe duda que, los llamados «Ejes Transversales», son uno de los pilares principales y soporte fundamentante, que alentaron la reforma educativa preconizada por la LOGSE. Se podría afirmar que, por primera vez, en nuestro sistema educativo se señalan y sistematizan, oficialmente, un conjunto de objetivos y contenidos educativos que transcienden los aspectos instructivos y cognoscitivos de la educación.

No es de extrañar que toda esta temática haya supuesto una cierta inquietud y desazón entre los actuales docentes, convirtiéndose en uno de los temas más polémicos y que mayor proliferación bibliográfica y documental está generando en el actual ámbito educativo, como puede verse, por citar sólo algunos, entre otros, los trabajos de M.E.C. (1993), Busquets (1993), Gervilla (1993), García Mínguez (1992 y 1994), González Lucini (1992, 1993 y 1994), Nieda (1992), Marchesi y cols. (1993), Fortuny (1994), Santos Rego y cols. (1994) Reyzabal (1995), ...

Parecen, pues, sobradas las razones que justifican el tema de este trabajo, deseando contribuir con él a plantearnos e intentar dilucidar, hasta donde nos sea posible, estos interrogantes: Después de unos años de implantación de la LOGSE: ¿Qué se está haciendo en los centros a propósito de los Temas Transversales? ¿Cómo se están llevando a la práctica? ¿Qué dificultades están surgiendo? ¿Cómo se programan y se abordan en los colegios? ... Si se trata de un aspecto altamente novedoso, diferencial y relevante, de nuestra actual normativa, bueno será que teóricos y prácticos de la educación unamos imaginación y esfuerzos para que los buenos deseos se conviertan en una realidad fecunda y creadora.

Como razones de fondo o justificativas de la transversalidad curricular el M.E.C. (1993), invoca la siguiente base argumental:

- Con la transversalidad se trata de ayudar a conseguir un modelo de persona desde una concepción profundamente humanista.

- Está determinada por situaciones socialmente problemáticas.

- Pretenden el desarrollo integral de la personalidad del alumnado

- Contribuyen a cultivar una dimensión ético-moral conectada con valores

- Deben procurar una estrecha relación escuela-entorno

- Deben estar presentes en todo el proceso educativo

- Abiertos a nuevas enseñanzas y necesidades del alumnado

La Transversalidad Curricular, por lo expuesto anteriormente, creemos que es un elemento y esfuerzo, plausible, para intentar trazar un horizonte educativo común para todos los ciudadanos que viven en una misma sociedad democrática, suponiendo unos objetivos formativos que impulsan y favorecen un desarrollo ético y unos comportamientos humanos y humanizadores, sin los cuales la sociedad no sólo no se desarrolla convenientemente sino que hasta corre el riesgo de empobrecerse o aniquilarse. La educación y la escuela no pueden ser insensibles a este encomiable objetivo, difícil de conseguir si los educadores no se lo toman muy en serio, dado que suponen...:

«Valores importantes tanto para el desarrollo personal e integral de los alumnos y alumnas, cuanto para un proyecto de sociedad más libre y pacífica y más respetuosa hacia las per- 
sonas y hacia la propia naturaleza que constituye el entorno de la sociedad humana», González Lucini(1994).

\section{Modalidad de tratamiento}

La problemática actual que la transversalidad está suponiendo en los centros creemos que no reside tanto, aunque también, en la sensibilidad hacia el tema como en la forma o modos de llevarla a la práctica o tratarla en la escuela.

Las estrategias para implementar la transversalidad se sintetizan, según Fortuny (1994) en las tres siguientes modelos:

«a) Modelo de ejes: Partir de los ejes transversales e integrar en ellos los contenidos de las distintas áreas o materias del curriculum, lo que supondría un planteamiento inverso a lo que viene haciéndose en la actualidad.

b) Modelo hábitos: Partir de un estudio de las necesidades del alumnado de un centro, contemplando sus hábitos y procedimientos en alguna temática concreta y a tono con ella establecer un plan de actuación que el profesorado, de una forma cooperativa, pondría en práctica.

c) Modelo de contenidos sobre un tema transversal: Partir del análisis de los posibles contenidos de un tema transversal, efectuado conjuntamente por todo el profesorado de un centro, con independencia de los diferentes niveles de formación o de especialidad y aportando cada uno sus puntos de vista, para seguidamente, efectuar una planificación y llevarla a la práctica entre todos».

Algunos autores, refiriéndose a las estrategias para llevar a cabo la transversalidad, lo mismo que ocurre, por ejemplo, con la educación para la carrera, Law (1981) habla de tres tipos de estrategias diferentes: Infusivas o de diseminación de los conceptos o temas a través de todas las materias o actividades escolares. Aditivas o como un programas más o curso a parte que se añade a los otros existentes. Mixtas o integrando la temática en determinadas áreas o materias.

Álvarez Rojo (1994), distingue cuatro modalidades diferentes, según que se trate de una adición, diseminación, infusión o inmersión de los contenidos transversales en el curriculum. Desde nuestro punto de vista, al menos se ofrecen tres posibilidades de alternativas para el tratamiento de los temas transversales. Una primera, que podríamos denominar $\boldsymbol{p a}$ ralela al curriculum. En esta modalidad, los temas transversales se tratarían de una forma no integrada en el curriculum, es decir, independiente del resto de las demás asignaturas y áreas curriculares. Como modalidad más frecuente sería dedicar alguna hora semanal al estudio de estos temas, por ejemplo, en una hora de tiempo libre, no lectiva, o de tutoría. El curriculum tendría sus momentos y los temas transversales el suyo propio, pero separados y sin ninguna interrelación. Es una modalidad frecuente que, posiblemente, para empezar el rodaje de esta nueva andadura, sea inevitable.

Otra modalidad de tratamiento, se podría denominar como integración parcial en el curriculum, ello supone un paso más con relación a la modalidad anteriormente descrita. Se trataría de abordar ciertos contenidos o aspectos de los temas transversales en el momento en que están relacionados con contenidos específicos del curriculum o con temas concretos. 
Por ejemplo, al dar en Ciencias el tema de la reproducción de los seres vivos, abordar aspectos, comunes y diferenciales de la sexualidad humana, referida a los niños, los adolescentes o los jóvenes. Se daría, pues, una cierta conexión e interrelación con algunos aspectos del curriculum oficial.

En tercer lugar, cabría hablar de una plena integración en el curriculum, refiriéndonos con ello a diseminar por las diferentes áreas del curriculum, sobre todo con aquellas que presenten una mayor afinidad, todos los temas transversales, tratándolos desde las diferentes disciplinas que integran la totalidad del curriculum. No cabe duda que ésta sería la forma mejor y más deseable de tratamiento pero, a la vez, la que mayores dificultades de coordinación, medios y tiempos requiere, dado que supone un planteamiento interdisciplinar con lo que ello comporta de coordinación, programación conjunta, etc. Esta modalidad puede ser viable en los primeros ciclos escolares pero muy discutible en los últimos o en secundaria

Siendo realistas y refiriéndonos a nuestro entorno, no creemos que sería demasiado afirmar que nuestros actuales centros se sitúan, la mayor parte de ellos, en la primera opción señalada y, tal vez, algunos, muy pocos, en la segunda de las alternativas descritas. La tercera modalidad, hoy por hoy, y en las actuales circunstancias, sería un bonito deseo pero actualmente irrealizable, como hemos tenido ocasión de comprobar al realizar recientemente un estudio empírico sobre el tema.

Deseamos insistir, una vez más, en que apoyamos el tratamiento de la transversalidad curricular desde su plena integración en el currículo, hasta donde ello sea posible. Sin embargo y porque sospechamos que no se está haciendo cuanto debería hacerse, dadas las dificultades que entraña, consideramos que un paso previo hasta que esto suceda, es realizar una intervención según el Modelo de Servicios pero mediante una intervención por programas, en el sentido en que definen esta modalidad de intervención, entre otros: Rodríguez Espinar y cols. (1993), Repetto (1994), Alvarez Rojo (1994) Bisquerra y cols. (1998), Vélaz de Medrano (1998), López Urquízar y col. (1999) Valls (1998) ...

En cualquier caso, e independientemente de la modalidad de tratamiento que se haga de la transversalidad, nos parecería que debería tener en cuenta unos mínimos requisitos en su articulación y programación para cualquier centro, como son:

- Realizar un análisis previo de las necesidades y de la realidad del contexto en que se encuentra un centro. No todos los contextos son iguales, con frecuencia acusan rasgos diferenciales que deben tenerse en cuenta, si de verdad se desea dar respuesta a problemas e inquietudes reales de los alumnos y de la sociedad. Por eso, junto a una Transversalidad Oficial, para todos, hay que situar otra, particular, de cada comunidad autónoma, ciudad o centro en concreto.

- Selección y jerarquización de las necesidades y problemas detectados. Es obvio que no todos los problemas son de la misma magnitud ni las necesidades detectadas presentan el mismo grado de intensidad o urgencia. Realizar un buen ordenamiento jerárquico que establezca prioridades de actuación puede ser un buen paso para la eficacia.

- Determinar los tópicos a afrontar. Definir los campos o el ámbito de actuación dentro de la transversalidad con el contenido que cada situación concreta requiere, parece también importante si no se desea correr el riesgo de llamar ejes transversales del curriculum a cualquier cosa. 
- Delimitación de los indicadores y manifestaciones específicos en los diferentes ciclos y niveles escolares. La edad del alumnado y el momento psicoevolutivo por el que está pasando condiciona el contenido y la forma de tratamiento de un tema transversal. No es igual tratar el problema de la sexualidad a los seis años, que a los doce, que a los diecisiete... Unos momentos escolares son más propicios para la prevención, otros para la detección y tratamiento, otros, incluso, para incluir medidas correctoras, ...

- Consenso en cuanto a las áreas de conocimientos que deben contribuir a afrontar un tema. Dependiendo de la temática a afrontar será preciso buscar la conexión con aquellas disciplinas o materias del curriculum, más idóneas y propicias, para que sean implicadas en un tratamiento interdisciplinar del tema.

- Programación específica. Articular la transversalidad no es otra cosa que equipararla en su tratamiento al resto de las materias curriculares, en las que se señalan objetivos de distinto tipo, contenidos, tiempos, duración, formas alternativas de tratamiento y evaluación, etc.

- Recursos y materiales adecuados. No se deberían afrontar los contenidos transversales como una materia más de la que es preceptivo examinarse y superarla. Reducirla a un libro de texto más, o a unos contenidos que hay que memorizar y aprender, podrían producir el efecto contrario al que se pretende. Es muy notable la cantidad y calidad de la producción audiovisual que está apareciendo referida a muchos de los temas transversales, saber seleccionar y disponer de una buena capacidad discriminativa puede ser una adecuada táctica para poner en juego una metodología lúdica, creativa, participativa, motivante, ...

- Evaluación. Han pasado los tiempos en que se creía que la evaluación debe limitarse a los conocimientos adquiridos por los alumnos. Una evaluación formativa -inicial o de proceso, criterial o normativa- debe contemplar, también, la viabilidad y funcionalidad de un programa si se quiere evitar cometer los mismos errores y rutinas a las que tan proclives somos los educadores. Los programas referentes a los ejes transversales deben ser evaluados para comprobar su eficacia. Máxime, si se tiene en cuenta que estamos iniciando una andadura que no debe ser viciada desde sus comienzos y que inevitablemente ofrece una complejidad muy resaltable, sobre todo, si se quiere hacer de una forma interdisciplinar y con el consenso y contribución de todo el profesorado que imparte las distintas áreas de conocimientos

\section{La «educación para la salud» en la transversalidad curricular}

Ya hemos afirmado que todos los temas transversales tienen una singular relevancia para el individuo y la sociedad. Esta es su razón de ser y su valor reside en conectar la educación con la vida. La salud, no cabe ninguna duda, es un bien y un valor en sí mismo de máxima transcendencia. Los países desarrollados, entre los que contamos al nuestro, han conseguido hacer de la salud un bien común, haciendo grandes esfuerzos y dedicando grandes medios y recursos a las prestaciones sanitarias. Hasta tal punto que entre nosotros, afortunadamente, todo ciudadano tiene una cobertura sanitaria prácticamente total. 
No obstante, aunque contamos como un logro importante del siglo veinte esta cobertura sanitaria con una amplia red de hospitales, médicos, material tecnológico altamente cualificado, sin embargo es preciso prestar una máxima atención a los aspectos preventivos de la salud. De tal forma que, bien podría afirmarse, que la calidad de un sistema sanitario es preciso medirla no tanto por su capacidad de curación como por la de prevención. De ahí las grandes campañas publicitarias y los grandes esfuerzos por concienciar a la población de aspectos higiénicos, sanitarios y alimenticios necesarios para evitar enfermedades de ulteriores y graves consecuencias, individuales y sociocomunitarias.

Si es cierto que los avances de la medicina en las últimas décadas han sido espectaculares, sin embargo, los riesgos de la salud siguen siendo muy importantes, a pesar de las grandes expectativas de vida con las que actualmente cuentan los países desarrollados. Sobre todo, existe una gran preocupación por la prevención de usos y costumbres que, a la larga, desembocan en problemas sanitarios de alcance colectivo y social. Ahí está ese creciente y amenazante mundo de la droga, del SIDA... que algunos no dudan en calificar como las modernas plagas del siglo $X X$. Por otra parte, son patentes y manifiestas las consecuencias de hábitos y costumbres de fuerte raigambre, como el consumo de alcohol y tabaco que, en opinión de los expertos, tantos problemas de salud están causando y que son hábitos y costumbres que se están iniciando en la adolescencia y juventud, cada vez a edades más tempranas, llegando a ser, con el tiempo, un grave obstáculo a superar dada la dependencia que suelen generar.

Bajo el epígrafe de «Educación para la Salud», en los temas transversales, suelen incluirse aquellos programas destinados a prevenir y a concienciar a los niños, adolescentes y jóvenes sobre el consumo de tabaco, alcohol, drogadicción, así como la prevención del SIDA, la evitación de embarazos no deseados en adolescentes y la educación vial, dado el número de accidentes de circulación que se están produciendo en la población juvenil, hasta tal punto que es la mayor causa de muerte entre la población menor de treinta años. Sin embargo, esto podría entenderse como un cierto reduccionismo, si se tiene en cuenta que la Educación para la Salud comprende un espectro bastante más amplio, como certeramente señala Fortuny (1994), al referirse a esta temática añadiendo otros tópicos como: Higiene y limpieza personal; actividad y descanso; desarrollo fisico; alimentación y nutrición; prevención de enfermedades; prevención de accidentes; salud y medio ambiente; salud mental; ...

Como no podía ser por menos, la escuela está siendo sensible a toda esta problemática y son frecuentes las campañas y programas que en ella se llevan a cabo, intentando hacer tomar conciencia a los niños y adolescentes sobre los factores de riesgo que actualmente pueden estar acechándoles. Tarea educativa no fácil ni exitosa a corto plazo, pero esperemos que provechosa a medio o largo plazo. Esta inquietud de la educación se está viendo reflejada en cantidad de publicaciones, monografías, sesiones tutoriales, charlas, conferencias, mesas redondas, vídeos, ... Valgan, a modo de muestra muy reducida y simplificada, las aportaciones y trabajos de Escamez (1990), Moron Marchena y cols. (1997), Sarlet (1996), Ronchon (1991), Busquets y cols. (1994) ...

Nuestro trabajo se inscribe dentro de esta inquietud y su razón de ser y motivación, más importante, es contribuir, en cuanto nos sea posible, a plantear estas preguntas nacidas de una corresponsabilidad, altamente sentida, como educadores: ¿Qué se está haciendo en los centros en esta línea? ¿Con qué preparación cuentan nuestros escolares en temas de tanta relevancia? 


\section{La Educación para la Salud}

La Educación para la Salud es una inquietud que desde hace algún tiempo va extendiéndose y logrando una gran penetración y resonancia en el actual tejido social, a impulsos de una serie de convicciones, acciones, programas y organismos, de muy diversa índole, cuya pretensión fundamental es lograr una sensibilización social hacia la salud, entendiéndola como un valor y derecho individual, social y comunitario que nos afecta a todos y en la que todos debemos sentirnos implicados.

Como hechos e instituciones propulsoras de esta sensibilización, en las últimas décadas, cabe señalar las contribuciones, que vienen realizando al respecto: La Unión Internacional para la Educación Sanitaria, organización mundial, no gubernamental, fundada en París en 1951; El Informe Lalonde de 1974; La Asamblea Mundial de la Salud: Perspectivas sociales en materia de salud, en 1976; Salud para todos en el año 2.000, OMS 1977); La Conferencia de Ata Alma, 1978; Conferencia Internacional sobre Promoción de la Salud, celebrada en Otawa, 1986; Conferencia de Adelaida, en 1988; Declaración de Liverpool sobre Derecho de la Salud, 1988; Conferencia Europea sobre Promoción de Educación para la Salud, Estrasburgo 1990; Conferencia de Sundsval, 1991; Conferencia de Educación Sanitaria, Helsinki, 1991; las Conferencias Mundiales de la Unión Internacional de Promoción y Educación para la salud (UIPES); ... Todos ellos organismos e iniciativas que mantienen bien encendida la antorcha de esta encomiable inquietud.

En el ámbito europeo, además de la Organización Mundial de la Salud (O.M.S.), cuya oficina europea reside en Copenhague y que viene desarrollando importantes programas sobre: Estilos de vida y salud; Promoción de la salud; Educación para la Salud; Ciudades Saludables; Salud Mental; Drogadicción; Prevención de alcoholismo; Tabaco y Salud; Nutrición; SIDA... son resaltables:

- El Consejo de Europa, con sede en Estrasburgo que dentro de su programa de trabajo incluye, entre otros, la educación y la sanidad. Su comité Europeo de Salud Pública, formado por los representantes de los Ministerios de Salud de los países miembros, se encarga de realizar programas, campañas, informes, congresos y encuentros, así como financiando proyectos relacionados con la educación y promoción de la salud

- La Unión Europea también viene ocupándose del tema ininterrumpidamente como puede verse en la Formulación de Recomendaciones sobre el tema, como la de 18-41988; o las Resoluciones del Consejo de 3-12-1990; 27-11-92; o el Tratado de Maastrich (7-2-1992) en el art. 129 se dice textualmente:

"La acción de la Comunidad se encaminará a la prevención de enfermedades especialmente de las más graves y ampliamente difundidas, incluida la toxicomanía, apoyando la investigación de su etiología y de su transmisión, así como la información y educación sanitaria.»

- En nuestro país pueden verse recogidas algunas de estas preocupaciones en materia de Educación Sanitaria en la normativa existente al respecto en:

- Constitución Española de1978: Art. 43: «Derecho a la Salud».

- La Ley General para la defensa de los Consumidores y Usuarios de 1984: Art. 18: Derecho a la Educación y formación del consumidor. 
- En la Ley General de Sanidad, de 1986, los Arts. 18,20,56,68, 70,73, se refieren a la promoción, protección y educación para la salud.

- Ley del Medicamento (1990): Capítulo III.

- Ley de Ordenación General del sistema Educativo, de 1990: Artículos 13,19,20 y 23. Se concibe la Educación Para la Salud como un tema transversal a incluir en el curriculum.

Son de considerar, además, las numerosas campañas publicitarias, cursos, publicaciones y programas que sobre Educación para la Salud vienen desarrollando una importante cantidad de organizaciones e instituciones, tanto públicas como privadas: Ministerio de Sanidad y Consumo, Consejerías de Salud y Bienestar de las diferentes Comunidades Autónomas o Ayuntamientos, Cruz Roja, ONGs, ... Todas ellas iniciativas y actividades que son una clara manifestación política, social y comunitaria por un tema de máxima trascendencia y alcances.

Hecha esta elemental referencia, es preciso hacer un primer intento de aproximación y clarificación del concepto de Educación para la Salud. Para ello creemos necesario, como lo hace Conde (1997), efectuar una cierta distinción entre unos cuantos términos muy afines y estrechamente relacionados:

- Promoción de la Salud: Es el proceso que tiene como objetivo capacitar al individuo y a la comunidad para que aumente el control sobre los determinantes de la salud (ambientales, económicos, políticos, individuales, ...).

- Prevención de la Salud: Conjunto de actuaciones diseñadas para reducir los factores generales de riesgo de enfermedad específica.

- Educación para la Salud: Proceso de comunicación interpersonal dirigido a proporcionar las informaciones necesarias para un examen critico de los problemas de salud, y a responsabilizar a los individuos y a los grupos sociales en las decisiones que tienen efectos directos o indirectos sobre la salud fisica y psíquica de las personas y de la colectividad. Es una herramienta esencial tanto para la prevención como para la promoción de la salud».

Sobre todo, es preciso distinguir, como bien señala Fortuny (1994), entre dos términos que suelen usarse indiferenciadamente pero que tienen connotaciones claramente diferentes: Educación para la Salud y Educación Sanitaria. Mientras que la segunda expresión hace referencia a la parcela educativa centrada en la enfermedad, con tildes claramente negativos, individuales, biológicos, reparadores o asistenciales, derivados de la enfermedad; la primera, en cambio, parte de una visión positiva por cuanto que se desprende de un concepto de salud más centrado en explicar en qué consiste la salud, positivamente, en lugar de preocuparse por lo que no es, es decir definir la salud por contraposición a la enfermedad.

Tomando como base la definición de la O.M.S., la Salud es entendida como el estado de completo bienestar físico, social y mental, así como la capacidad de funcionar en la sociedad y no sólo la ausencia de enfermedad.

Puede verse, cómo con el paso del tiempo, el concepto de salud se ha ido modificando. Hoy día está clara la necesidad de contemplar la salud en términos positivos, dinámicos, holísticos y ecológicos, en los que la educación, la participación comunitaria y los aspectos sociales juegan un importante papel, Morón (1997). Con lo que el concepto de Educación para la Salud ha experimentado una importante evolución, alejándose un tanto de lo que 
originariamente se entendió por tal. Dentro de esta perspectiva parece oportuno reflejar aquí algunos de los conceptos de salud más al uso en el actual contexto social. Veamos algunas de las más frecuentes y representativas:

«Si conceptualizamos la salud individual no como un estado que se posee independientemente de la conducta del sujeto, sino como un proceso dinámico y constructivo que, mediante la puesta en práctica de recursos propios y ajenos, tiende al logro del equilibrio bio-psico-social y permite el desarrollo humano en sintoníal armonía con el ambiente, es decir un proceso de aprendizaje adaptativo, concluiremos en la necesidad que tienen las personas de intervenir responsablemente en la adquisición, defensa, promoción y reconstrucción de su salud».

En la misma línea se pronuncia Sainz Martín (1997) al afirmar:

«La Educación para la Salud, como aprendizaje no sólo aspira a conseguir hábitos que fomenten la salud, sino que intenta favorecer una filosofia más vitalista, natural y humana del hombre moderno, intenta pues que éste tenga más libertad y que dicha libertad sea conquistada por el aumento de conocimientos y formas de vida más responsables, tanto los individuos (para sí y para los otros) como las colectividades».

Este mismo autor señala que la Educación para la Salud, puede ser considerada como:

«Un proceso de orden intelectual, psicológico y social que comprende actividades destinadas a incrementar la aptitud de los individuos a tomar decisiones-con conocimiento de causa-que afectan a su bienestar personal, familiar y social».

Con una mayor concisión pero con total claridad se puede expresar el concepto de Educación para la Salud que expone Morón (1997):

"Cuando hablamos de Educación para la Salud nos referimos a un instrumento de promoción que proporciona estrategias para que los sujetos deseen lo más saludable cuando el entorno les permite elegir entre varias opciones». El mismo autor, abundando en lo dicho se expresa así: "La Educación para la Salud, de manera simplificada, puede definirse como una actividad que pretende aportar elementos a los individuos y a la comunidad para que se modifiquen los comportamientos hacia conductas más saludables».

De particular interés puede resultar el concepto del MEC (1993: 17), al señalar que:

«La Educación para la Salud se plantea como todas aquellas actuaciones encaminadas a favorecer en las personas una manera de pensar, de sentir y de comportarse que desarrolle al máximo su capacidad de vivir-individual y colectivamente- en equilibrio con su entorno fisico, biológico y sociocultural».

De las definiciones y conceptos expuestos, se deduce que no existe una definición homogénea y comúnmente aceptada por todo el mundo, dándose connotaciones muy peculiares de unos autores y países a otros. Pero se dan unas bases bastante comunes y generalizadas en la concepción de la Educación para la Salud que bien podrían expresarse en los términos siguientes:

- Necesidad de propugnar la concienciación del Derecho a la Salud de toda persona.

- Necesidad de reducir los principales problemas de salud a que estamos expuestos todos los ciudadanos de las actuales sociedades. 
- Necesidad de combatir hábitos, costumbres y usos perjudiciales

- Necesidad de impulsarla desde la infancia

- Debe ser una educación holística, global e integral.

- Debe basarse, sobre todo, en la prevención.

- Debe tener como soporte una política pública, saludable, que contenga todos los aspectos implicados (legislación, medidas fiscales y económicas ...).

- Debe fomentar y crear ambientes saludables.

- Debe participar e implicarse toda la comunidad.

- Debe desarrollar hábitos y actitudes personales que tienen mucho que ver con diferentes medios y contextos: familia, escuela, sociedad.

- Debe abarcar como contenidos fundamentales: Información con base científica, concienciación responsable y cambio de actitudes o hábitos perjudiciales.

- La salud está influida por los siguientes determinantes: La biología humana (herencia, crecimiento y envejecimiento): el medio ambiente (contaminación del aire, agua, alimentos, ruido, ...); los hábitos de vida (estilos y pautas individuales de conducta); la organización de los servicios de salud (actuaciones preventivas, curativas y rehabilitadoras proporcionadas por los agentes sanitarios.

\section{La Escuela y la Educación para la Salud}

Estamos convencidos de que algunos de estos graves problemas, incluidos en lo que hemos definido como educación para la salud, como: la drogadicción, el tabaquismo, el alcoholismo, etc. no se curan con medidas policiales o represivas sino con medidas educativas, en las que la escuela tiene un decisivo papel que jugar. Por ello, la educación no puede permanecer de espaldas a unos problemas que son un peligro y un riesgo que comienza a edades tempranas. Existe una gran denuncia social generalizada de que cada vez es más precoz el inicio en el consumo de estas sustancias, de tal forma que la población escolar es un sector claro de riesgo para su consumo, tan perjudicial para la salud como para iniciarse en unos peligrosos hábitos que, posteriormente, son muy difíciles de erradicar. De ahí el papel preventivo que debe jugar la escuela y los educadores en una dimensión tan preocupante como lo es la salud humana (Vega Fuente, 1983).

En el concepto de Educación para la Salud, es muy frecuente encontrar en los diferentes autores consultados, como puede comprobarse por lo más arriba expuesto, concebirla como un proceso de aprendizaje, Morón (1997) Sainz Conde (1997) ... Es decir, la salud es susceptible de ser potenciada y mejorada mediante el aprendizaje. Si alguna institución esta estrechamente relacionada con el aprendizaje es la escuela. Y ello por una doble razón: Porque en ella se encuentran las personas en trance de formación, en un período de edad muy receptivo, sensible e influenciable por los estímulos ambientales; y porque la escuela debe procurar aprendizajes funcionales y vivenciales, válidos para la vida y la adaptación al medio.

Otras razones podrían invocarse como la transmisión a los niños, adolescentes y jóvenes, de valores y actitudes, constructivos y potenciadores, entre los que cabe destacar como pertenecientes a un primer rango, se parta de la orientación axiológica que se quiera, los re- 
lacionados con la salud por ser uno de los más importantes, en ausencia de los cuales la vida se percibe como muy limitadora y frustrante. Por lo cual la escuela y los sistemas educativos no pueden desentenderse, en modo alguno de los aspectos relacionados con la salud.

En la convicción de que la escuela puede ser un medio educativo para la salud de primera magnitud, ha ido surgiendo todo un movimiento cuyo empeño es proporcionar una adecuada Educación para la Salud, nos referimos a las Escuelas Promotoras de Salud.

La denominación de Escuelas Promotoras de Salud fue acuñada por la O.M.S., representando una clara filosofía de la concepción de los centros educativos favorecedores de hábitos saludables, como medios eficaces para promover el intercambio de hábitos saludables, como un medio eficaz para estimular la adquisición de experiencias e información, así como la difusión de prácticas adecuadas relacionadas con la salud. Estas escuelas se agrupan en una Red Europea llevada a cabo por tres estamentos del máximo prestigio: La Oficina Regional Europea de la OMS, la Comisión de la Unión Europea y el Consejo de Europa.

La filosofía derivada de las Escuelas Promotoras de Salud es, en expresión de Morón (1997), asimilable por cualquier centro escolar, dado que los objetivos y características con las que se autodefinen las Escuelas Promotoras de Salud (OMS, 1995), se corresponden con unos principios y objetivos que dicho autor formula así:

- Favorecer modos de vida sanos y ofrecer al alumnado y al profesorado opciones, a la vez realistas y atractivas, en materia de salud.

- Definir objetivos claros de promoción de la salud y de seguridad para el conjunto de la comunidad escolar.

- Ofrecer un marco de trabajo y de estudio dirigido a la promoción de la salud, donde se tengan en cuenta las condiciones del edificio escolar, de los espacios deportivos y de recreo, los comedores escolares, los aspectos de seguridad de los accesos, ...

- Desarrollar el sentido de responsabilidad individual, familiar y social en relación con la salud.

- Posibilitar el pleno desarrollo físico, psíquico y social y la adquisición de una imagen positiva de sí mismo, en todo el alumnado.

- Favorecer buenas relaciones entre todos los miembros de la comunidad educativa y de ésta con su entorno.

- Integrar la Educación para la Salud en el Proyecto Curricular, de forma coherente, utilizando metodologías que fomenten la participación del alumnado en el proceso educativo.

- Proporcionar al alumnado los conocimientos y habilidades indispensables para adoptar decisiones responsables en cuanto a su salud personal y que contribuyan al desarrollo de la salud y seguridad de su entorno.

- Identificar y utilizar los recursos existentes en la colectividad para desarrollar acciones a favor de la promoción de la salud.

- Ampliar la concepción de servicios de salud escolar para hacer de ellos un recurso educativo que ayude a la comunidad a utilizar adecuadamente al sistema sanitario.

Quienes defienden la necesidad de introducir la Educación para la Salud en la escuela, se apoyan en los siguientes argumentos justificativos: 
1. La escuela incide en la vida de los individuos durante un período psicoevolutivo en que se aprende a tomar decisiones: También las referidas a la salud.

2. El periodo de escolarización se caracteriza por una gran flexibilización, propicia para adquirir hábitos y actitudes positivas. Las edades tempranas son más receptivas y pueden aprenderse en ella hábitos y comportamientos perdurables a lo largo de toda la vida.

3. Es muy importante tomar medidas educativas preventivas en la escuela para no tener que adoptarlas posteriormente correctivas y terapéuticas.

4. El que en muchos países la práctica totalidad de la población infantil y adolescente esté escolarizada, es una forma de llegar a tiempo a la totalidad de los ciudadanos.

5. La escuela no puede volver la espalda a los grandes problemas sociales que afectan al individuo, sino que debe procurar aprendizajes funcionales y conectados con la vida y la realidad social.

\section{La cruda realidad}

Ya hemos afirmado con anterioridad que, en la práctica escolar, el problema fundamental consiste en cómo, cuándo y con qué afrontar la temática transversal. Nosotros creemos que el problema es de tal transcendencia que no se puede abordar de cualquier forma, es preciso subrayar la conveniencia de abordar esta temática con una modalidad de intervención por programas o de servicios pero interviniendo por programas.

Los estudiosos y expertos en programas de intervención orientadora o psicopedagógica, señalan como características que definen las señas de identidad de un programa de orientación las siguientes:

- El programa se basa en unos adecuados principios teóricos, claros y bien delimitados y justificados.

- El programa supone una diacronía y secuencia de planificación que discurre por las siguientes fases o etapas: Definición del problema $\rightarrow$ análisis del contexto $\rightarrow$ identificación, valoración y priorización de necesidades $\rightarrow$ definición de metas y objetivos $\rightarrow$ diseño del programa $\rightarrow$ aplicación o implementación del programa $\rightarrow$ evaluación del programa.

- El programa está contemplado en el Proyecto Curricular del Centro y sometido a su programación general.

- Tiene un carácter fundamentalmente preventivo y de desarrollo más que curativo, remedial o terapéutico.

- El programa se estructura por objetivos.

- En el programa intervienen todos los agentes de la comunidad educativa compartiendo responsabilidades y tareas tendentes a un mismo fin: la eficacia.

- La evaluación formativa y retroalimentadora es una inquietud que precede, acompaña y finalizada con el programa comprobando tanto su funcionamiento como su eficacia.

- El programa tiene en cuanta tanto el proceso como los resultados. 
- El programa supone la secuenciación de objetivos, contenidos, actividades, criterios de evaluación, asignación de medios y recursos.

- Se basa en las necesidades de los sujetos.

- Al alumno se le concede una participación activa y un protagonismo compartido.

- Suele tener una cierta transcendencia sociocomunitaria.

- Etc.

En abierto contraste con lo dicho, mucho nos tememos que las formas de intervención en la transversalidad, en general, y en la educación para la salud en particular, los actuales centros educativos distan mucho de lo que sería teóricamente deseable. Así parece afirmarlo nuestra experiencia al realizar otro trabajo, Carballo y cols. (2000)para el que fue preciso ponerse en contacto una docena de centros, públicos y privados. En esta toma de contacto, de una forma un tanto informal se preguntaba al profesorado cómo estaban tratando la transversalidad, encontrándonos con la desagradable sorpresa de que se estaba haciendo poco o nada al respecto, por más que la normativa lo mandara. En el mejor de los casos, se estaban llevando a cabo intervenciones esporádicas, puntuales, informales y casi siempre basadas en la buena voluntad de algunos tutores o de algunos colectivos altruistas y humanitarios.

En honor a la verdad, y sin ánimo de denunciar a nadie, pudimos comprobar una sospecha que no hubiéramos deseado confirmar: Los temas transversales estaban siendo reducidos, en la mayor parte de los centros, a un bonito deseo, pero acusándose una lamentable ausencia de planteamientos serios y básicos para su tratamiento de una forma sistemática y rigurosa. Los profesores y tutores consultados estaban sensibilizados con el tema considerándolo necesario y urgente, sin embargo no se sentían capacitados para actuar o no contaban con los momentos adecuados para ello. Por otra parte, conversaciones, también informales, con un considerable número de orientadores escolares o psicopedagogos de la E.S.O., así como con profesores de diferentes tipos de colegios y niveles educativos se manifestaban un tanto pesimistas y negativos sobre lo que los centros estaban haciendo con uno de los que se consideró pilar fundamental de la actual normativa nacida de la LOGSE.

Lo peor de este importante problema no es que no se esté abordando la temática con la seriedad y rigor con que debería hacerse, sino que, además, no parece que los centros tengan conciencia de que es una omisión, a nuestro parecer grave, de imponderables consecuencias. No es de extrañar que los medios de comunicación ante problemas tan recientes como los del «botellón», el consumo de alcohol, tabaco, drogadicción, ... nos estén recriminando a los educadores qué es lo que estamos haciendo en la escuela. Esta dolorosa acusación es la que nos obliga, a más de una década ya de distancia de la puesta en práctica de la transversalidad curricular, a replanteárnosla y preguntarnos qué se está haciendo de ella, reivindicando una urgente, efectiva y eficaz presencia de ella en los centros educativos.

\section{Referencias bibliográficas}

Álvarez González, M. (1991): «La evaluación en los programas de Orientación: evaluación del contexto y del diseño». Revista de Investigación Educativa, Vol. 9, nº 17, pp. 49-82.

Álvarez González, M. (1995): Orientación Profesional. Barcelona: Cedesc. 
Álvarez Martín, N. (1994) Los transversales. MEC. Dirección Provincial de Cantabria.

Álvarez Rojo, V. (1993): «Ámbitos temáticos y opciones metodológicas de la investigación en el campo de la Orientación». En Revista de Orientación Educativa y Vocacional,4 (5), pp. 11-23.

Andrés Orizo, F. (1991): Los Nuevos Valores de los Españoles. La Encuesta Europea de Valores en España. Madrid: S. M. - Fundación Santa María.

Arasa Ferrer, H. (1991): Educación para la salud: propuestas para su integración en la escuela. Zaragoza: Departamento de Sanidad.

Bisquerra Alzina, R. (1998): Modelos de Orientación e Intervención Psicopedagógica. Barcelona: Praxis.

Bolivar Botia, A. (1992): Los contenidos actitudinales en el curriculum de la Reforma. Madrid: Escuela Española.

BREZINKA, W. (1990): La Educación en una sociedad en crisis. Madrid: Narcea.

BUSQUETS, M y otros (1994): Los temas transversales. Claves de la formación integral. Madrid: Santillana.

Calvo Bruzos, S. (1991): Educación para la salud en la escuela. Madrid: Díaz de Santos.

Carballo, R. Fernández, M. J. y García Nieto, N. (2000): «Eficacia de un programa de apoyo y refuerzo de habilidades y aprendizajes instrumentales en niños con dificultades escolares». En Revista Bordón, Volumen 52; núm. 4.

Conde Huelva, A.M. (1997): «Programa comunitario de Educación para la Salud». En Morón, J. A., Salud, educación y sociedad. Sevilla: Diputación de Sevilla/Ayuntamiento de Dos Hermanas.

Costa, M. y López, E. (1986): Salud Comunitaria. Barcelona: Martínez Roca.

Donati, P. P. (1994): Manual de sociología de la salud. Madrid: Díaz de Santos.

Elzo Imaz, J. y otros (1994): Jóvenes Españoles. Madrid: Fundación Santa María.

Escamez, J. y otros (1986): La enseñanza de actitudes y valores. Valencia: Nau-Llibres.

Escamez, J. (1990): Drogas y escuela. Una propuesta de prevención. Madrid: Dykinson.

Escamez, J. y otros (1993): Educación para la Salud. Valencia: Nau Llibres - Generalitat Valenciana.

Fernández Sierra, J. y otros (1992): Evaluación cualitativa de programas de Educación para la salud. Granada: Aljibe.

Fortuny, M. (1994): «El contenido transversal de la educación para la Salud: diseño de la acción pedagógica». En Revista Ciencias de la Educación, n 159 , Julio-Septiembre de 1994.

García Mínguez, J. (1992): Los temas transversales como criterio de calidad educativa. Granada: Proyecto Sur.

García Mínguez, J. (1994): «Los ejes transversales del curriculum. Una aproximación a la calidad educativa». En Revista de Ciencias de la Educación, núm. 157.

Gervilla Castillo, E. (1993): «Los valores en la L.O.G.S.E. Enseñanzas de régimen general». En Rev. Bordón, Vol. 45; núm. 4 (pp. 435-446).

González Lucini, F. (1992): «Educación en valores y diseño curricular». En García Mínguez, J.: LOGSE: Perfiles para una nueva educación. Granada, I.C.E.

González Lucini, F. (1994a): Temas transversales y áreas curriculares. Madrid: Anaya.

González Lucini, F. (1994b): Temas transversales y educación en valores. Madrid: Alauda-Anaya.

Gysbers, N. C. y Henderson (1988): Developing and Managing Your School Guidance Program. Alexandria, Virginia: AACD.

Gysbers, N. C. (1990): Comprehensive Guidance programs that work, University of Michigan, School of Education, ERIC/Counseling and Personnel Services Cleringhouse. 
López, M. L. y otros (1995): «La institucionalización de los Servicios de Orientación Vocacional». En F. Rivas (Ed.) Manual de Asesoramiento y Orientación Vocacional. Madrid: Síntesis, pp. 159162.

López Urquizar, N. y otros (1999): Orientación Escolar y tutoría. Granada: Grupo Editorial Universitario.

Marchesi, A. y otros (1993): Los temas transversales en el curriculum. Madrid: Alauda.

Manzanares Gavilán, M. (1993): Educación para la salud en la formación inicial de maestros. Editor: Córdoba.

Manzano Soto, N. (1999): Elaboración y validación de un sistema de indicadores para evaluar programas de Orientación Educativa. Tesis doctoral (inédita) Madrid: Universidad Complutense de Madrid.

M.E.C. (1992): Materiales para la reforma. Infantil. Transversales. Madrid: M.E.C.

M.E.C. (1992): Materiales para la reforma. Primaria. Transversales. Madrid: M.E.C.

M.E.C. (1993): Temas transversales y desarrollo curricular. Madrid: Dirección General de Renovación Pedagógica - MEC.

M.E.C. y Ministerio de Sanidad y Consumo (1993): Tabaquismo y alcoholismo. Madrid: Dirección General de Renovación Pedagógica - MEC/ Colección de materiales didácticos de Educación para la Salud.

M.E.C. (1995): Transversales. Educación para la salud. Educación sexual. Madrid: Ministerio de Educación y Ciencia.

Morón Marchena, J. A. (Dir.) (1997): Salud, Educación y Sociedad. Sevilla: Diputación de SevillaAyuntamiento de dos Hermanas

Nieda, J. (1992): Transversales. Educación para la salud. Educación sexual. Madrid: Ministerio de Educación y Cultura.

O.M.S. (1978): Conferencia Internacional de atención Primaria de la Salud. Declaración de Alma Ata. U.N.I.C.E.F., URSS.

O.M.S. (1986): Carta de Otawa para la promoción de la Salud. Otawa.

O.M.S. (1995): Promoción de la salud de la Juventud Europea. La Educación para la salud en el ámbito educativo. O.M.S.

Ordaña, J. R. (1992): Educación para la salud en Enseñanza Secundaria: SIDA. Guía del educador. Murcia: Consejería de Sanidad.

Ortega Murcia, J. y otros (1993): Materiales didácticos. Educación para la salud. Tabaquismo y Alcoholismo: un problema social. Madrid: Ministerio de Sanidad y Consumo.

Pascual, M. A. (1988): Clarificación de valores y desarrollo humano. Madrid: Narcea.

Polaino Lorente, A. (1987): Educación para la Salud. Barcelona: Herder.

Repetto, E. (1994): Modelos actuales de Programas para el desarrollo de la Carrera. Desarrollo de la Carrera: Modelos y Programas Actuales. AEOEP. Valencia, pp. 19-33.

Reyzabal, M. J. y Sanz, A. (1995): Los ejes transversales. Aprendizaje para la vida. Madrid: Escuela Española.

Rodríguez Diéguez, A. (1992): «Precisiones conceptuales en torno al Career Education». En Actas de las VI Jornadas ACO-EP, Barcelona.

Rodríguez Espinar, S. (1992): «Orientación y reforma: el reto de la intervención por programas». Revista Qurriculum, Vol. 5, pp. 27-47. 
Rodríguez Espinar, S. (Coord.) (1993): Teoría y práctica de la Orientación Educativa. Barcelona: PPU.

Rodríguez Moreno, Mª L. (1992): Enseñar y aprender a tomar decisiones vocacionales. Madrid: M.E.C.

Ronchon, A. (1991a): Educación para la salud. Lleida: Pagés Editors.

Ronchon, A. (1991b): Educación para la salud. Guía práctica para realizar un proyecto. Barcelona: Masson.

Sainz Martín, M. (1997): «La juventud de la cultura de la salud». En Morón, J. A. (Coord.), Salud, educación y sociedad. Sevilla: Diputación de Sevilla/Ayuntamiento de Dos Hermanas.

Santos Rego, M. (1994): «Transversalidad y Educación». En Revista Ciencias de la Educación, no 159, Julio-Septiembre de 1994.

Sanz Oro, R. (1990): «El diagnóstico pedagógico desde la perspectiva de programas de intervención en Orientación Educativa». Revista Bordon, Vol. 42, $\mathrm{n}^{\circ} 1$.

Sanz Oro, R. (1996): Evaluación de programas en Orientación Educativa. Madrid: Pirámide (2ºdición).

Sarlet Gerken, A. M. (1996): Salud, Medio-Ambiente, Tolerancia y Democracia: la transversalidad educativa. Barcelona: PPU

Serrano González, M. I. (1990): Educación para la salud y participación comunitaria. Una perspectiva metodológica. Madrid: Díaz de Santos.

Valls Fernández, F. (1998): Fundamentos de Orientación Profesional para Psicopedagogos. Almería: Servicio de Publicaciones de la Universidad de Almería.

Vargas, A. y Palacios, V. (1993): Educación para la salud. México: McGraw-Hill.

Vega Fuente, A. (1983): Los educadores ante las drogas. Madrid: Santillana.

Vega Fuente, A. (1984): Los maestros y las drogas (Programa de formación básica). Bilbao: Mensajero.

Velaz de Medrano, C. (1998): Orientación e Intervención Psicopedagógica: Concepto, modelos, programas y evaluación. Granada: Aljibe.

Ventura, M. (1992): Actitudes, valores y normas en el currículo escolar. Madrid: Escuela Española.

Fecha de recepción: 05-11-02

Fecha de revisión: 10-10-03

Fecha de aceptación: 27-10-03 